\title{
Effects of selected noises on the quantum memory of satellite based quantum repeaters
}

\author{
András Mihály and László Bacsárdi, Member, IEEE
}

\begin{abstract}
Quantum repeaters are a key part of long-range free-space quantum key distribution. They allow us to circumvent the negative effects of the no-cloning theorem. Quantum repeaters are also a key point in point-to-point communication since otherwise, a direct line of sight would be necessary. In our simulation, we examined the QKD capabilities of quantum repeaters in a satellite-based network, along with selected types of noises.
\end{abstract}

Index Terms-QKD, Quantum memory, amplitude damping, dephasing noise, depolarizing noise, quantum communications

\section{INTRODUCTION}

$\mathbf{R}$ SA-based public-key cryptography is a part of our everyday life. It's used by banks, websites, and every other entity that wants to ensure its communications are secure on the web. But with the advancement of quantum computers and thanks to Shor's algorithm [1], the time when publickey-based encryptions will be broken is at our doorstep. By utilizing quantum computing, we can not only break today's most used encryptions, but we can also speed up the problemsolving for various problems. Using Groover's algorithm, we can find a record in unordered data in $\sqrt{N}$ time [2], or even extreme values [3]. By utilizing quantum computing we can solve problems like multi-user detection [3] or optimal resource distribution [4]. Quantum key distribution (QKD) is a subpart of quantum communication that can not only alleviate these problems thanks to the symmetric key-based encryption it's enabling, but (if used right) is virtually unbreakable. One problem with QKD using systems is the no-cloning theorem, which entails that we cannot copy a quantum bit.

This problem can be mitigated with the use of entanglement swapping quantum repeaters. To perform this task, we use the side effect of the Bell state measurement (BSM), which we detail later in the paper in section IV. But for these operations, quantum repeaters use two critical elements: quantum logic gates and quantum memories. Which are both affected by various noises.

Today's research is mostly connected to quantum satellites that use only one in their simulation, for example, the Chinese Micius [5], the Canadian QEYSSat [6], or the European QKDSat [7]. In our research, we simulated not a single satellite but a network of quantum satellites.

The authors are with the Department of Networked Systems and Services, Budapest University of Technology and Economics, Budapest, H-1117, Hungary.

E-mail: andras.mihaly.1998@gmail.com,bacsardi@hit.bme.hu.

This research was supported by the Ministry of Innovation and Technology and the National Research, Development and Innovation Office within the Quantum Information National Laboratory of Hungary. L. Bacsárdi thanks the support of the János Bolyai Research Scholarship of the Hungarian Academy of Sciences.
In our simulation of satellite networks composed of multiple quantum repeaters, the repeaters were simulated with varying amplitude damping, depolarizing, and dephasing noises affecting quantum memory. Our goal was to identify which noise types could be the most damping for a future quantum satellite network. So that we know what types of noise reductions are crucial for quantum memories. We also simulated multiple network setups, from real-world point-to-point (eg. BudapestMoscow) to varying node-to-node distance and node cardinality.

The structure of the paper is as follows: in Section II. we detail the current state of quantum key distribution. After that, we present the available selection of quantum network simulators. In Section IV. we discuss the use of satellite-based quantum repeaters and their edge over fiber-based networks. Then, we present our simulation and its architecture on the module level. In Section VI, we present our findings on the effects of various noise on the error rate.

\section{QUANTUM KEY DISTRIBUTION TODAY}

QKD despite, being a relatively new technology, already has multiple uses. One of the most important applications of quantum technology is quantum cryptography. Using quantum key distribution protocols like BB84 [8] or E91 [9], we can create secure sources of communication with the combination of one-time padding and symmetric-key encryption.

Quantum protocols are versatile, for example we can take the aforementioned protocols E91 and BB84. While the protocol developed by Charles and Gilles in 84 (hence the name) uses the quantum randomness of the photon polarization for its algorithm. On the other hand, in the development of the E91 QKD protocol Artur used quantum entanglement as means to generate secure key bits. There are also variations of protocols, for example, SARG04 [10] is derived from the BB84 QKD protocol but made for weak laser pulses instead of single photons. Today when we are talking about quantum-key distribution medium, we can consider two different platforms for quantum key distribution medium.

Fiber-based $Q K D$ : using fiber, we have a controllable medium at the cost of forcing the network to only used the established links. To date, there have been multiple experiments utilizing fiber-based QKD. To name a few: the first quantum key distribution over $48 \mathrm{~km}$ distance in Los Alamos [11] or the first quantum key distribution using a commercial fiber network in china [12].

Free-Space $Q K D$ : using free-space communicational channel (either terrestrial or satellite-based), we gain the ability 
Effects of selected noises on the quantum memory

of satellite based quantum repeaters to change established links and gain even lower the error rate originating from the medium by using satellites to relay our quantum bits. Until nowadays, there have been multiple experiments regarding the use of satellites in quantum key distribution. The first satellite-relayed intercontinental quantum network between Viena and Beijing [13], and the first entanglement-based quantum key distribution using a satellite at the altitude of $1,200 \mathrm{~km} \mathrm{[14],} \mathrm{are} \mathrm{one} \mathrm{of} \mathrm{these.}$

With the evolution of quantum satellites and communication, the dependency on different types of optical communications became to play an important role in free-space and satellite communications [15] [16] .

A detailed explanation of QKD is out of scope for this paper, but an in-depth survey can be found in [17].

\section{Quantum Satellite Networks}

As shown in Section II, using fiber as our primary medium for QKD can lower the number of possible connections. Using satellite-based quantum repeater networks has multiple advantages over the aforementioned fiber-based counterpart. Using a single satellite can already increase our coverage area depending on the elevation of the said satellite. The only negative drawback is the increase in latency since no information can travel faster than light. To negate this, we used an array of satellites at lower $(500 \mathrm{~km})$ altitudes. But since we are using a multitude of quantum repeaters, the errors that arose from different noises can increase along with the increased number of nodes or increased distance.

It is important to note that in this paper we don't use the error rate generated by the different types of aerial turbulences. A paper detailing the various effects of atmospheric turbulence and earth-satellite degree can be found in [18].

In our simulation, for the satellite positions, we used the data gathered from the Starlink satellite network. The use of Starlink satellites was self-evident since they mostly operate in low earth orbits and are working in large groups for increased coverage.

A quantum satellite repeater network would not only allow secure point-to-point communication, but they also would be able to create a secure network for communication, that uses QKD for encryption. In this paper, we analyze the effects of 3 types of noises (dephasing, depolarizing, and amplitude damping) on the quantum memory of quantum repeaters. Using various simulations, we simulated networks along with various noise rate combinations and routes, with differing node-to-node distance and node cardinality.

\section{QUANTUM SIMULATORS}

In our research, we tried multiple quantum network simulators before ending up with NetSquid. The three main simulators that we tried are Simulaqron [19], Squanch [20] and Netsquid [21].

Simulaqron is developed by QuTech and provides an application-level simulation of quantum networks. Since our simulation needed low-level simulation capabilities, we did not choose this one.
Squanch although provides an almost hardware-level simulation, didn't have the capability to also work on a higher level as Netsquid.

Finally, our search stopped with Netsquid which is also being developed at QuTech and provided us with all the needed features, from programable quantum processors to massive networks with multiple nodes.

\section{OUR SETUP}

\section{A. Overview of our simulator}

The simulator uses the Netsquid framework and the quantum repeater example as a base. The simulator can be divided into four sub-modules as it is illustrated in Fig 1

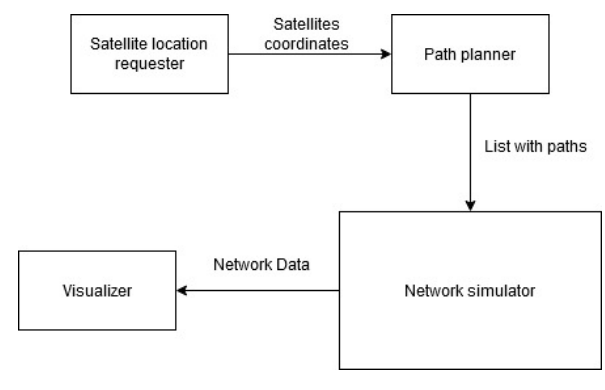

Figure 1. The architecture of the simulator

Satellite location requester: using N2YO.com's API [22], this module requests the location of all STARLINK satellites for the next $\mathrm{N}$ seconds $(N<300)$

Path planner: using Dijkstra's algorithm, the module calculates the optimal route between two terrestrial nodes.

Network simulator: simulates the quantum entanglement exchange beside varying noises.

Visualizer: using the output data from the network simulator module, depicts the change in quantum bit error rate (QBER). The node architecture setup follows the Fig. 1. A quantum source supplies entangled quantum bits to the local and nextin-line node's quantum processor. Using that, the quantum processor executes the Bell state measurement swapping the entanglement between quantum bits. The simulator runs multiple times with varying levels of amplitude damping, dephasing, and depolarizing noise. The chance to depolarize calculated using the following formula:

$$
\begin{array}{r}
P_{\text {depolarization }}=1-\exp (-\operatorname{delay}[\mathrm{ns}] * \\
\text { depolarization rate } \left.[\mathrm{Hz}] * 10^{-9}\right)
\end{array}
$$

For dephasing, the chance to occur is is calculated with the following formula:

$$
\begin{array}{r}
P_{\text {dephasing }}=1-\exp (-\operatorname{delay}[\mathrm{ns}] * \\
\text { dephasing rate } \left.[\mathrm{Hz}] * 10^{-9}\right)
\end{array}
$$

In the case of amplitude damping, instead of probability, the formula gives us the damping parameter $\gamma$.

$$
\gamma=1-\exp \left(\text {-delay[ns] } * \text { amplitude damping } * 10^{-9}\right)
$$


$\gamma$ is used in the following quantum operation:

$$
e(p)=\sum_{i} E_{i} p E^{\dagger}
$$

Where $E_{i}$ is substituted with the corresponding matrix from the equations found in 5, 6, 7 and 8

$$
\begin{gathered}
E_{0}=\sqrt{p}\left[\begin{array}{cc}
1 & 0 \\
0 & \sqrt{1-\gamma}
\end{array}\right] \\
E_{1}=\sqrt{p}\left[\begin{array}{cc}
0 & \sqrt{\gamma} \\
0 & 0
\end{array}\right] \\
E_{2}=\sqrt{1-p}\left[\begin{array}{cc}
\sqrt{1-\gamma} & 0 \\
0 & 1
\end{array}\right] \\
E_{3}=\sqrt{1-p}\left[\begin{array}{cc}
0 & 0 \\
\sqrt{\gamma} & 0
\end{array}\right]
\end{gathered}
$$

\section{B. Overview of our topology}

In our simulation, the topology we used was a linear one. As seen in Figure 2, the nodes have two crucial elements:

The quantum CPU is responsible for the execution of BSM on the qubits got from the two inputs (Quantum input 0, Quantum Input 1). The quantum source function is the generation of entangled quantum bits, one for the local quantum CPU and the other for the next node.

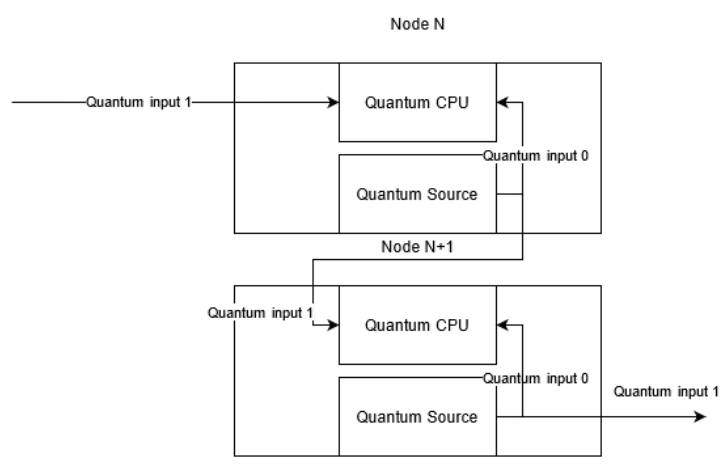

Figure 2. The simulated topology

\section{Quantum repeater composition}

Quantum repeaters [23] are the key to quantum communication over long distances. The repeaters utilize the consequence of Bell state measurement, which is swapping entanglements. For instance, we have two entangled quantum bit pairs, namely $Q_{11}-Q_{12}$ and $Q_{21}-Q_{22}$. After applying BSM on $Q_{11}$ and $Q_{22}$, we get two new entangled pairs: $Q_{11}-Q_{22}$ and $Q_{12}-Q_{21}$, as observed, a quantum entanglement generates between $Q_{12}-Q_{21}$ without the need for physical contact. With BSM, we can generate entangled quantum bits over large distances[23]. In our simulation, we generated entanglement between two terrestrial nodes by using quantum repeaters mounted on satellites. For the quantum entanglement swapping, we also require quantum memory, in this paper, we focused on how various quantum memory noises affect the quantum bit error rate.

\section{RESUlTS}

In our paper, we made three types of simulations:

Budapest-Moscow: the simulation was made with real data from satellites over the region, simulating a network of quantum repeaters.

Iterative increase of the distance: this simulation was made specifically to examine the effects of increasing distances on QBER.

Iterative increase of the number of nodes: the simulation was made to test the effects of increasing the number of nodes on QBER.

\section{A. Budapest-Moscow trajectory}

The simulation produced interesting results: the different types of noises yielded different values of QBER with the same distance. To validate these claims, we created the other two simulation variants.

As in the next section, we can see. The increase in the distance affected the QBER differently depending on the type of noise. For example, in the case of depolarizing noise, the error rate stayed well under 0.5 for most of the simulations.

\section{B. Iterative increase of distance}

In this simulation, we examined the effects of distance increases on the QBER for the sake of the experiment the number of nodes stayed at a constant value of 5 . As expected and seen in Fig. 3, 4, and 5 the error rate increased along with the distances. At the maximum distance of $12200 \mathrm{~km}$, almost all noise rates produced a QBER of 0.5, with the sharpest increase along the dephasing rate. The first time each noise combination reached the QBER value of 0.5 is visible in the aforementioned figures. The distance values for each iteration can be using equation 9:

$$
D_{i}=2800+i * 600 \text { and for every } \mathrm{i} \equiv 1 \bmod 4 \rightarrow+200
$$

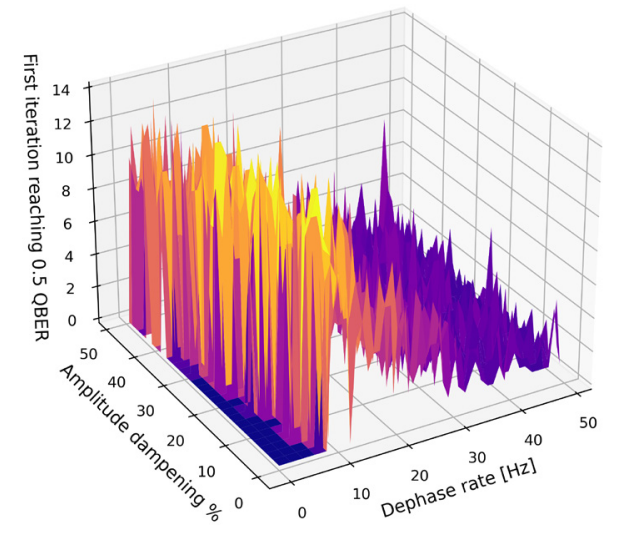

Figure 3. The first iteration of the simulation that reached a QBER of 0.5 with dephasing and amplitude damping noises. The exact distance can be calculated by using the equation 9 . 
Effects of selected noises on the quantum memory

of satellite based quantum repeaters

The figure 3 details the first time an iteration reached the error rate of 0.5 for each noise combination. On the Z-axis, we can see the iteration on a scale from 0 to 14 , and on the $X$ and $\mathrm{Y}$ axes, we can see the error rates. As previously mentioned, and seen in figure 3 , in the case of distance, the most crucial element is the dephasing noise, which reached the QBER of 0.5 as soon as the second iteration.

\section{Iterative increase of the number of nodes}

In the case of iterative node volume increase, we can see that in the case of this simulation, had a similar effect on the QBER as the iterative increase in distance, which we can see in Fig. 6, 7, and 8. The slight variation that we can observe on the figures is thanks to the variability of the simulator. The number of hops for each iteration is available in Table I. Looking at the aforementioned figures, we can discover the same pattern as in the one with an iterative increase in distances.

Table I

TABLE CONTAINING THE NUMBER OF HOPS FOR EACH ITERATION OF THE SIMULATOR

\begin{tabular}{|l|l|l|l|l|l|l|l|l|l|} 
Iteration: & 0 & 1 & 2 & 3 & 4 & 5 & 6 & 7 & 8 \\
Hops: & 5 & 6 & 7 & 7 & 8 & 9 & 9 & 10 & 11 \\
Iteration: & 9 & 10 & 11 & 12 & 13 & 14 & & & \\
Hops: & 11 & 12 & 13 & 13 & 14 & 15 & &
\end{tabular}

\section{CONCLUSION}

In this paper, we presented the effects of various noises on the quantum memory of quantum repeaters and their impacts on the quantum bit error rate. We could see that from the amplitude damping, dephasing, and depolarizing trio, the most susceptible to the increase due to distance and node number was the dephasing noise (as seen in Sections VI. B. and VI. C). In the end, we can conclude that for future satellite networks, one of the most crucial noises is the quantum dephasing noise, and in the future, we should prioritize minimizing it.

In this paper, we only examined one element of the quantum repeaters with one type of error model. In the future, we would like to rerun the simulation with different error models to test the correlation between the iterative distance and node number increases mentioned before in Section V. C.

Another angle for future research is to introduce new variables in our simulation. The two models we would like to use to expand our simulation are aerial turbulences and quantum gates.

\section{APPENDIX}

\section{A. Figures}

First time reaching 0.5 QBER

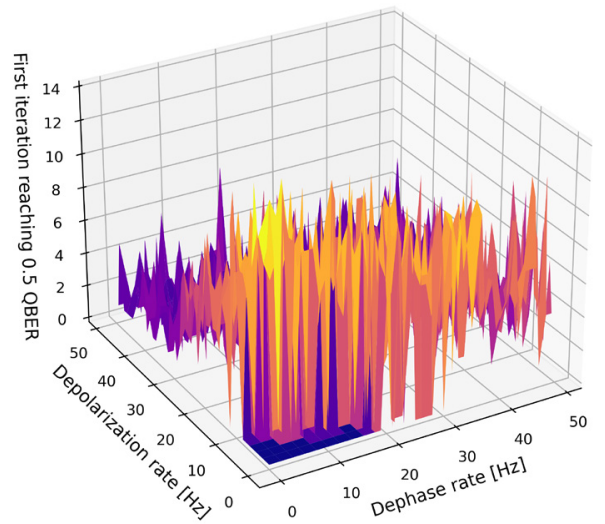

Figure 4. The first iteration of the simulation that reached a QBER of 0.5 with dephasing and depolarizing noises. The exact distance can be calculated by using the equation 9 .

First time reaching 0.5 QBER

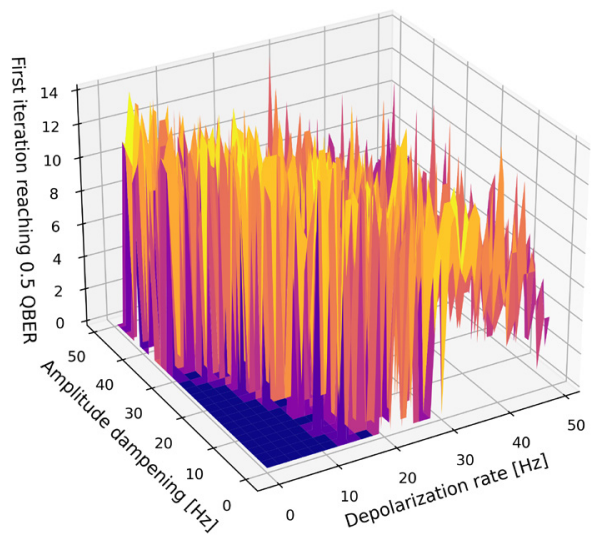

Figure 5. The first iteration of the simulation that reached a QBER of 0.5 with depolarizing and amplitude damping noises. The exact distance can be calculated by using the equation 9 . 
Effects of selected noises on the quantum memory satellite based quantum repeaters

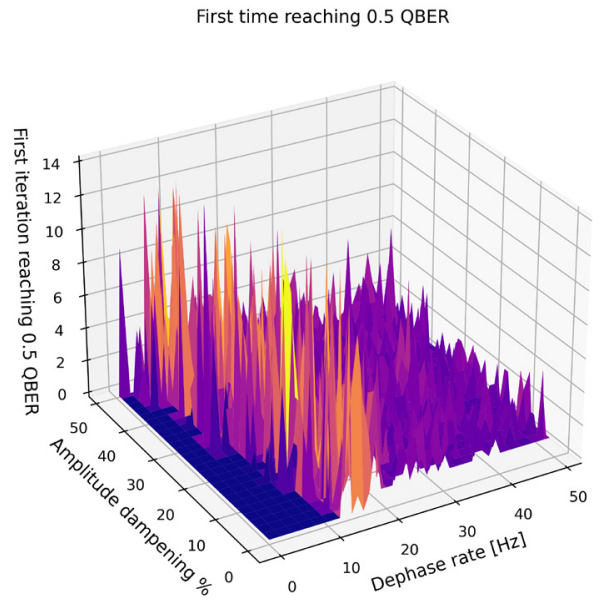

Figure 6. The first iteration of the simulation that reached a QBER of 0.5 with dephasing and amplitude damping noises. With varying hops and a constant distance of 6200 . The number of hops can be seen in the table I.

First time reaching 0.5 QBER

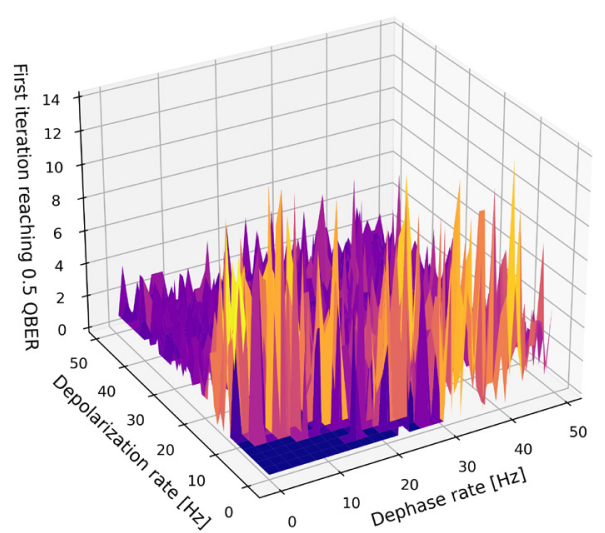

Figure 7. The first iteration of the simulation that reached a QBER of 0.5 with dephasing and depolarizing noises. With varying hops and a constant distance of 6200 . The number of hops can be seen in the table I.

First time reaching 0.5 QBER

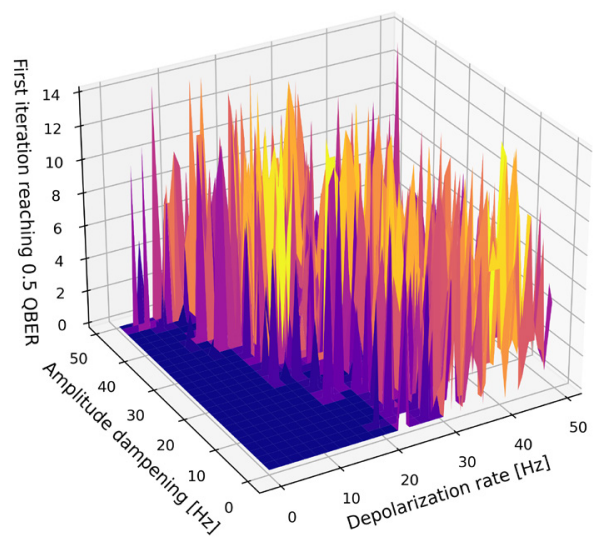

Figure 8. The first iteration of the simulation that reached a QBER of 0.5 with depolarizing and amplitude damping noises. With varying hops and a constant distance of 6200 . The number of hops can be seen in the table I.

\section{ACKNOWLEDGEMENT}

L. Bacsárdi thanks the support of the János Bolyai Research Scholarship of the Hungarian Academy of Sciences.

\section{REFERENCES}

[1] Peter Shor. "Algorithms for Quantum Computation: Discrete Logarithms and Factoring". In: Proceedings of 35th Annual Symposium on Foundations of Computer Science (Oct. 1996). DoI: 10.1109/SFCS.1994.365700.

[2] Lov Grover. "From Schrödinger's equation to the quantum search algorithm". In: Pramana-journal of Physics - PRAMANA-J PHYS 56 (Feb. 2001). DoI: 10.1007/s12043-001-0128-3.

[3] S. Imre. "Quantum Existence Testing and Its Application for Finding Extreme Values in Unsorted Databases". In: IEEE Transactions on Computers 56 (2007). DoI: 10.1109/TC.2007.1032.

[4] Sara Gaily and Sándor Imre."Quantum Optimization of Resource Distribution Management for Multi-Task, Multi-Subtasks". In: Infocommunications journal 11 (Jan. 2019), pp. 47-53. DOI: 10.36244/ICJ.2019.4.7.

[5] Shengkai Liao et al. "Satellite-Relayed Intercontinental Quantum Network." In: Physical review letters 1203 (2018), p. 030501. DoI: 10.1103/PhysRevLett.120.030501.

[6] Canadian Space Agency. Quantum Encryption and Science Satellite (QEYSSat). Oct. 2020. URL: https://www.asc-csa.gc.ca/eng/satellites/ qeyssat.asp.

[7] Secure communication via quantum cryptography. URL: https://www. esa.int/Applications/Telecommunications_Integrated_Applications/ Secure_communication_via_quantum_cryptography.

[8] Charles H. Bennett and Gilles Brassard. "Quantum cryptography: Public key distribution and coin tossing". In: Theoretical Computer Science 560 (Dec. 2014), pp. 7-11. ISSN: 0304-3975. DOI: $10.1016 /$ j.tcs.2014.05.025.

[9] Artur Ekert. "Ekert, A. K.: Quantum Cryptography Based on Bell's Theorem. Phys. Rev. Lett. 67(6), 661”. In: Physical review letters 67 (Sept. 1991), pp. 661-663. DoI: 10.1103/PhysRevLett.67.661.

[10] Cyril Branciard et al. "Security of two quantum cryptography protocols using the same four qubit states". In: Phys. Rev. A 72 (3 Sept. 2005), p. 032301. DoI: 10.1103/PhysRevA.72.032301.

[11] Richard J. Hughes, George L. Morgan, and C. Glen Peterson. "Quantum key distribution over a $48 \mathrm{~km}$ optical fibre network". In: Journal of Modern Optics 47.2-3 (2000), pp. 533-547. DOI: $10.1080 / 09500340008244058$.

[12] Yichen Zhang et al. "Continuous-variable QKD over $50 \mathrm{~km}$ commercial fiber". In: Quantum Science and Technology 4.3 (May 2019), p. 035006. DoI: 10.1088/2058-9565/ab19d1.

[13] C. S. N. Koushik et al. "A Literature Review on Quantum Experiments at Space Scale-QUESS Satellite". In: Innovations in Electronics and Communication Engineering. Ed. by H. S. Saini et al. Singapore: Springer Singapore, 2020, pp. 13-25. DOI: 10.1007/978-981-15-3172-9_2.

[14] Juan Yin et al. "Entanglement-based secure quantum cryptography over 1,120 kilometres". In: Nature 582 (June 2020), pp. 1-5. DOI: $10.1038 / \mathrm{s} 41586-020-2401-y$.

[15] Andrea Farkasvolgyi and Istvan Frigyes. "Optical transfer in space communication”. In: Infocommunications Journal 10 (Sept. 2018), pp. 9-13. DOI: 10.36244/ICJ.2018.3.2.

[16] Eszter Udvary. "Visible Light Communication Survey". In: Infocommunications journal (Jan. 2019), pp. 22-31. DOI: 10.36244/ICJ.2019.2.3.

[17] Laszlo Gyongyosi, Laszlo Bacsardi, and Sandor Imre. "A Survey on Quantum Key Distribution”. In: Infocommunications journal (Jan. 2019), pp. 14-21. DoI: 10.36244/ICJ.2019.2.2.

[18] Mate Galambos and Laszlo Bacsardi. "Comparing Calculated and Measured Losses in a Satellite-Earth Quantum Channel". In: Infocommunications Journal 10 (Sept. 2018), pp. 14-19. DOI: 10.36244/ICJ.2018.3.3. 
Effects of selected noises on the quantum memory of satellite based quantum repeaters

[19] A. Dahlberg and S. Wehner. "SimulaQron - A simulator for developing quantum internet software". In: ArXiv/abs/1712.08032 (2017).

[20] Ben Bartlett. "A distributed simulation framework for quantum networks and channels". In: arXiv:1808.07047 (2018).

[21] Tim Coopmans et al. "NetSquid, a discrete-event simulation platform for quantum networks". In: arXiv preprint arXiv:2010.12535 (2020).

[22] N2YO.com API, N2yo.com, 2021. [Online]. Available: https://www.n2yo.com/api/. [Accessed: 02-Feb-2021].

[23] Sandor Imre and Laszlo Gyongyosi. Advanced quantum communications: an engineering approach. Wiley-Blackwell, 2013.

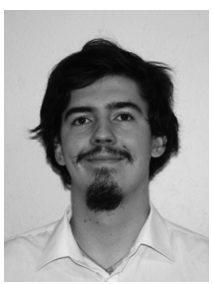

András Mihály studied at Göllner Mária Regional Waldorf secondary school, received his BSc degree, and started his MSc education in 2021 in Computer Engineering from the Budapest University of Technology and Economics (BME). In 2020 he reached 3rd place in the local Scientific student conference. Currently, he is pursuing research in the quantum field.

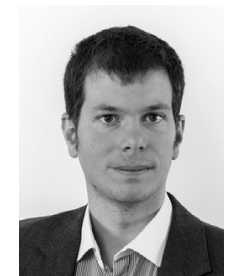

László Bacsárdi (M’07) received his MSc degree in 2006 in Computer Engineering from the Budapest University of Technology and Economics (BME) and his $\mathrm{PhD}$ in 2012. He is corresponding member of the International Academy of Astronautics (IAA). Between 2009 and 2020, he worked at the University of Sopron, Hungary in various positions including Head of Institute of Informatics and Economics. Since 2020, he is associate professor at the Department of Networked Systems and Services, BME and head of Mobile Communications and Quantum Technologies Laboratory. His current research interests are quantum computing, quantum communications and ICT solutions developed for Industry 4.0. He is chair of the Telecommunications Chapter of the Hungarian Scientific Association for Infocommunications (HTE), Vice President of the Hungarian Astronautical Society (MANT). Furthermore, he is member of AIAA, IEEE and HTE as well as alumni member of the UN established Space Generation Advisory Council (SGAC). In 2017, he won the IAF Young Space Leadership Award from the International Astronautical Federation. 